\title{
Renal ultrasonographic shear-wave elastography and urinary procollagen type III amino-terminal propeptide in chronic kidney disease dogs
}

\author{
Chutimon Thanaboonnipat ${ }^{1}$ (D), Saikaew Sutayatram² ${ }^{2}$, Chollada Buranakarl² ${ }^{(\mathbb{D})}$ and Nan Choisunirachon $^{1}$ (i)
}

1. Department of Veterinary Surgery, Faculty of Veterinary Science, Chulalongkorn University, Bangkok, 10330, Thailand; 2. Department of Veterinary Physiology, Faculty of Veterinary Science, Chulalongkorn University, Bangkok, 10330, Thailand.

Corresponding author: Nan Choisunirachon, e-mail: nan.c@chula.ac.th

Co-authors: CT: chutimon.th@chula.ac.th, SS: saikaew64@hotmail.com, CB: chollada.b@chula.ac.th

Received: 01-05-2020, Accepted: 04-08-2020, Published online: 23-09-2020

doi: www.doi.org/10.14202/vetworld.2020.1955-1965 How to cite this article: Thanaboonnipat C, Sutayatram S, Buranakarl C, Choisunirachon N (2020) Renal ultrasonographic shear-wave elastography and urinary procollagen type III amino-terminal propeptide in chronic kidney disease dogs, Veterinary World, 13(9): 1955-1965.

\begin{abstract}
Background and Aim: Renal fibrosis is a well-established pathological alteration associated with chronic kidney disease $(\mathrm{CKD})$ in several species and progresses as CKD advances. Although a renal biopsy is the gold standard for determining renal fibrosis, it is an invasive, impractical method for clinical practice. In humans, ultrasonographic shear-wave elastography (SWE), a novel advanced diagnostic imaging tool, can evaluate renal parenchyma stiffness, and urinary procollagen type III amino-terminal propeptide (uPIIINP), a promising renal fibrosis biomarker in humans, has increasingly been use applied to reduce the biopsies. This study compares renal tissue elasticity observed through SWE Young's modulus (E) values between healthy dogs (HD) and those with CKD.

Materials and Methods: The E value acquired by SWE, uPIIINP levels, and renal function were evaluated in 15 CKD dogs and $15 \mathrm{HD}$.

Results: The renal cortical E values were significantly higher than the renal medullary E values in both groups ( $\mathrm{p}<0.001)$. Renal cortical and medullary E values in CKD dogs were significantly higher than in $\mathrm{HD}(\mathrm{p}<0.01)$. Cortical $\mathrm{E}$ values had greater significant correlations with renal functional parameters than the medullary E values and had a significant positive correlation with concentrations of plasma creatinine $(\mathrm{Cr})(\mathrm{p}<0.001)$; blood urea nitrogen $(\mathrm{p}<0.01)$; urine protein $\mathrm{Cr}$ ratio $(p<0.01)$; and fractional excretions of sodium $(p<0.05)$, potassium $(p<0.05)$, chloride $(p<0.05)$, and magnesium $(p<0.001)$ while they had a negative correlation with urine specific gravity $(\mathrm{p}<0.05)$ and urine osmolality to plasma osmolality ratio $(\mathrm{p}<0.05)$. The uPIIINP to $\mathrm{Cr}(\mathrm{uPIIINP} / \mathrm{Cr})$ ratios of CKD dogs were higher than those of HD ( $<0.001)$. Moreover, the uPIIINP/Cr levels presented significant correlations with the renal cortical E values $(\mathrm{p}<0.01)$ and also the renal functional parameters.
\end{abstract}

Conclusion: SWE offers a complementary, non-invasive diagnostic imaging tool for evaluating renal tissue stiffness in CKD dogs with renal function deterioration. In addition, uPIIINP levels are associated with renal function and structural changes in dogs. Therefore, the uPIIINP level might be a non-invasive, complementary, and promising biomarker for evaluating renal fibrosis in canine CKD.

Keywords: chronic kidney disease, dog, renal fibrosis, shear-wave elastography, urinary procollagen type III amino-terminal propeptide.

\section{Introduction}

Chronic kidney disease (CKD), the most common renal disorder in dogs, is an irreversible and progressive impairment of kidney structure or function from permanent injury that has exceeded the maximal capacity of compensation, lasting for at least 3 months [1-4]. CKD encompasses a wide variety of kidney problems either congenital or acquired problems, of which the initiating cause of the CKD usually cannot be identified $[1,3]$. The commonly reported causes of canine CKD

Copyright: Thanaboonnipat, et al. Open Access. This article is distributed under the terms of the Creative Commons Attribution 4.0 International License (http://creativecommons.org/licenses/ by/4.0/), which permits unrestricted use, distribution, and reproduction in any medium, provided you give appropriate credit to the original author(s) and the source, provide a link to the Creative Commons license, and indicate if changes were made. The Creative Commons Public Domain Dedication waiver (http:// creativecommons.org/publicdomain/zero/1.0/) applies to the data made available in this article, unless otherwise stated. include incomplete recovery from an acute renal injury, pyelonephritis, glomerulonephritis, tubulointerstitial disease, neoplasia, hypercalcemia, and various hereditary nephropathies [1-5]. The prevalence of canine CKD in the United Kingdom was reported to be approximately $0.5-1.5 \%$ [5]. Demographic predisposing factors of canine CKD consist of advancing age [6-8], male sex [2], and breed including Chinese Shar-Pei [9], Bull Terrier [10], English Cocker Spaniel [11], West Highland White Terrier [12], Boxer [13], and mixed breeds [2]. Renal fibrosis, especially tubulointerstitial fibrosis, is an irreversible and progressive pathological change reported to occur with CKD in both humans and dogs [14-17] and is a main pathological change during the CKD advancement $[16,17]$, subsequently leading to impairment of renal functions. Therefore, early diagnosis facilitating early treatment is important to slow CKD progression [18-20]. Early clinical signs of canine CKD 
are usually non-specific [3]. Although measuring the glomerular filtration rate (GFR) is considered the gold standard for evaluating renal function, methods for GFR assessment are technically cumbersome and not practical for clinical implementation [21]. The blood creatinine $(\mathrm{Cr})$ concentration is commonly used for screening impaired renal function. However, $\mathrm{Cr}$ is a non-specific marker, because it can be affected by various factors, including sex and muscle mass [21-23]. B-mode ultrasonography is a common diagnostic imaging modality that can be used to evaluate anatomical changes in the kidneys, including size, shape, and contour. Notably, the normal ratio of renal length to the aortic luminal diameter $(\mathrm{K} / \mathrm{Ao})$ in normal dogs ranges from 5.5 to 9.1 [24], while this ratio is lower in dogs with advanced CKD [25].

Many renal fibrosis biomarkers are currently used to evaluate renal fibrosis in humans, but there is still no precise method that can replace a renal biopsy $[26,27]$. However, a renal biopsy is an invasive procedure with several complications; therefore, it is not performed in routine clinical practice, particularly in high-risk patients such as dogs with CKD [28]. Urinary procollagen type III amino-terminal propeptide (uPIIINP) has been developed as a promising renal fibrosis biomarker in humans [16,29]. The uPIIINP has been reported to be one of promising biomarkers in humans that can indicate the degree of renal fibrosis and CKD progression $[16,30,31]$. In veterinary medicine, it has recently been reported that the uPIIINP to $\mathrm{Cr}$ (uPIIINP/Cr) ratio was significantly higher in cats with CKD than in healthy cats [32]. Furthermore, the plasma PIIINP levels have been utilized as a fibrosis marker for assessing cardiac remodeling [33], idiopathic pulmonary fibrosis [34], and liver fibrosis in dogs [35]. In addition, the advanced diagnostic imaging techniques, particularly renal ultrasonographic shear-wave elastography (SWE), have been increasingly performed in humans [36,37]. Increases of both cortical and medullary renal echogenicity detected by B-mode ultrasound are related to the CKD lesions [38]; these increases may also be present in dogs without evidence of renal impairment [39]. SWE can evaluate renal parenchyma elasticity or renal tissue stiffness $[40,41]$ by facilitating the Young's modulus (E) value measurements [42]. The previous human and feline studies have shown that renal $\mathrm{E}$ values of humans and cat CKD patients are significantly higher than those of healthy persons and cats $[32,37]$; moreover, the renal $\mathrm{E}$ values also correlate with decreases in renal function in CKD patients $[32,36]$.

In veterinary medicine, the information about the uPIIINP biomarker in CKD dogs has not been previously reported and the SWE information is currently only available in dogs with normal kidneys [43].

Therefore, the aims of this study were, first, to compare the renal tissue elasticity observed through E values, acquired by SWE, between healthy dogs (HD) and CKD dogs; second, this study evaluated the relationships between the renal tissue stiffness and functional renal parameters, including the plasma $\mathrm{Cr}$ concentration, blood urea nitrogen (BUN), urine specific gravity (USG), the urine protein $\mathrm{Cr}$ (UPC) ratio, and the urine osmolality to plasma osmolality (Uosm/Posm) ratio as well as, the fractional excretion of sodium, potassium, chloride, and magnesium ( $\mathrm{FE}_{\mathrm{Na}}$, $\mathrm{FE}_{\mathrm{K}}, \mathrm{FE}_{\mathrm{Cl}}$, and $\mathrm{FE}_{\mathrm{Mg}}$ ) in both groups. Third, this study compared uPIIINP levels between HD and CKD dogs and determined the relationship between $\mathrm{UPIIINP} / \mathrm{Cr}$ ratios and renal $\mathrm{E}$ values.

\section{Materials and Methods}

\section{Ethical approval and informed consent}

This study was approved by the Chulalongkorn University Animal Care and Use Committee, Faculty of Veterinary Science, Chulalongkorn University (Protocol number: 1731055) and all participating dog owners signed consent forms. This study was performed within a visit day and then all client-owed dogs were allowed to go back home.

\section{Study location and period}

This study was performed using client-owned dogs that presented to the Small Animal Teaching Hospital, Faculty of Veterinary Science, Chulalongkorn University between September and December 2017.

\section{Animals and experimental design}

The dogs which age-matched were assigned to either the HD (HD; $n=15)$ or CKD dogs (CKD; $n=15)$ groups. Dogs with normal findings based on history, physical examination results, and routine blood examinations, including complete blood counts $(\mathrm{CBC})$ and plasma biochemistry, USG and UPC ratio as well as abdominal radiography, and B-mode abdominal ultrasound were considered to be HD. CKD dogs were defined based on the International Renal Interest Society (IRIS) staging system that defined CKD based on a blood Cr level of more than $1.4 \mathrm{mg} / \mathrm{dl}$ for more than 3 months; these levels were considered stable on being considered for inclusion [44]. All CKD dogs were subjected to the same diagnostic procedures as the HD group. Dogs presenting any signs of ascites, infectious diseases, lymphoma, or congenital kidney diseases, such as polycystic kidney, nephrolith, and hydronephrosis were excluded from this study.

On the day of the visit, the blood samples were collected from all dogs in tubes containing either of ethylene diamine tetra-acetic acid (EDTA) (K3 EDTA collection test tube, FL MEDICAL, Italy) for CBC measurement or lithium heparin (VACUETTE ${ }^{\circledR}$, Greiner Bio-One $\mathrm{GMbH}$, Germany) for determining the concentrations of $\mathrm{Cr}, \mathrm{BUN}$, alanine aminotransferase (ALT), alkaline phosphatase (ALP), total protein, and albumin. Some of the collected plasma was centrifuged and stored at $-20^{\circ} \mathrm{C}$ for analysis of plasma osmolality and electrolytes $\left(\mathrm{Na}^{+}, \mathrm{K}^{+}, \mathrm{Cl}^{-}\right.$, and $\left.\mathrm{Mg}^{2+}\right)$. Microscopic observation of urine sediment was performed before measuring urine protein levels, to 
exclude canine patients with urinary tract infections. In addition, urine samples were collected and stored at $-20^{\circ} \mathrm{C}$ for evaluations of the concentrations of $\mathrm{Cr}$, protein, PIIINP, and electrolytes $\left(\mathrm{Na}^{+}, \mathrm{K}^{+}, \mathrm{Cl}^{-}\right.$, and $\mathrm{Mg}^{2+}$ ) concentrations as well as osmolality. Indirect systolic blood pressure was measured in triplicate at the proximal hind limb or distal forelimb in each dog, and the average value was used.

\section{Analytical procedures}

Plasma and urine concentrations of $\mathrm{Cr}, \mathrm{BUN}$, ALT, ALP, total protein, and albumin were analyzed by an automated analyzer (The IL ILab 650 Chemistry Analyzer, Diamond diagnostic, USA). Frozen plasma and frozen urine samples $\left(-20^{\circ} \mathrm{C}\right)$ were thawed to measure the osmolality and electrolytes concentrations. Posm and Uosm were measured by an automatic cryoscopic osmometer (OSMOMAT ${ }^{\circledR}$ 030, Gonotec $\mathrm{GmbH}$, Germany). Plasma and urine electrolyte concentrations were determined by an automated machine (ARCHITECT c16000 clinical chemistry analyzer, Abbott Laboratories Ltd, USA) and an automated analyzer (COBAS INTEGRA ${ }^{\circledR} 400$ plus, Roche Diagnostics Ltd., Switzerland), respectively. USG was determined using a refractometer (Master Refractometer, $\mathrm{ATAGO}^{\circledR}$, Japan). The urine protein level was determined after protein precipitation with $3 \%$ sulfosalicylic acid [37]. The $\mathrm{FE}_{\mathrm{Na}}, \mathrm{FE}_{\mathrm{K}}, \mathrm{FE}_{\mathrm{Cl}}$, and $\mathrm{FE}_{\mathrm{Mg}}$ were calculated as previously described [45]. Systolic indirect blood pressure was measured using a Doppler blood pressure instrument (Vet-Dop 2 ${ }^{\mathrm{TM}}$, Vmed Technology, USA). The concentrations of uPIIINP were determined in frozen urine samples $\left(-80^{\circ} \mathrm{C}\right)$ in duplicate using a double sandwich enzymelinked immunosorbent assay (ELISA) kit (Canine Procollagen Type III N-Terminal Propeptide (PIIINP) ELISA Kit, Cat. No. MBS2608207; MyBioSource, USA), following the manufacturer's instructions. The intra-essay and inter-essay coefficient of variation were $<12 \%$. The uPIIINP/Cr ratios were calculated and expressed as $\mathrm{ng} / \mathrm{mgCr}$.

\section{B-mode ultrasonography and SWE procedure}

The ultrasonographic examinations were performed without any sedation. Dogs were manually restrained and positioned in either right or left lateral recumbency for each ipsilateral renal observation. Hair was clipped and acoustic gel was applied to the skin in preparation for the ultrasound procedure. B-mode ultrasonographic images were obtained using a $9 \mathrm{MHz}-$ bandwidth of linear transducer (Resona-7, Mindray Medical International, China) to screen the overall abdominal organs including the kidney. The K/Ao ratio was assessed by measuring the maximal length of each kidney, in the sagittal plane, as well as the aortic luminal diameter, as previously described [24]. All measurements were performed twice, and the results were then averaged for analysis. Then, SWE was examined by the same instrument on the sagittal plane of the kidney. This instrument uses the Sound Touch
Elastography technology that an elastogram displays as a dual-screen mode of a B-mode image and a color quantitative elastogram. The elastogram expresses a red color to represent the hardest tissues whereas a blue color represents the softest tissues. During the SWE procedure, a transducer is carefully placed over the kidney with a small amount of pressure to maintain high reliability (RLB). The RLB map indicates the SWE image quality, presented as percentages, and elasticity results from the high RLB of shear-wave images are more reliable. The fixed region of interest was adjusted as wide as possible to cover both renal cortex and renal medulla. The Young's modulus (E) value in kilopascal unit was selected to represent renal parenchyma elasticity; a high $\mathrm{E}$ value indicates stiff parenchyma, while lower E value signifies soft parenchyma $[42,46]$. Three separated the elastograms were selected and renal $\mathrm{E}$ values were measured at the nearest to a probe and central renal parenchyma using a circular electrical caliper with the same size at the renal cortex and medulla in both of HD and CKD dogs (Figure-1); finally, then renal $\mathrm{E}$ values were averaged.

\section{Statistical analysis}

All data analyses were performed using GraphPad Prism $7^{\circledR}$ software (GraphPad Software, CA, USA). The results are expressed as mean \pm standard deviations. The normality of the data distribution was tested with the Shapiro-Wilk test. The average $E$ values between the left and right kidneys in each group were analyzed by paired t-test. The average E values and uPIIINP/Cr ratios were compared between groups using the unpaired t-test or Mann-Whitney test. Pearson's correlation coefficient and Spearman's correlation coefficient were used to investigate the correlations between parameters. Statistical significance was considered if $\mathrm{p}<0.05$.

\section{Results}

The geographic data of HD and CKD groups are presented in Table-1. The mean ages of both groups in this study were age-matched. The HD group consisted of Shih Tzu (7), Yorkshire Terrier (3), Siberian Husky (1), Cardigan Welsh Corgi (1), Cocker Spaniel (1), Poodle (1), and Chihuahua (1), while the CKD group encompassed Shih Tzu (5), Chihuahua (3), Mixed breed (3), Siberian Husky (1), American

Table-1: Demographic information of HD and CKD dogs.

\begin{tabular}{lcc}
\hline Parameters & $\begin{array}{c}\text { HD group } \\
(\mathbf{n = 1 5})\end{array}$ & $\begin{array}{c}\text { CKD group } \\
(\mathbf{n = 1 5})\end{array}$ \\
\hline Sex & & \\
$\quad$ Female (spayed) & $10(1)$ & $4(2)$ \\
$\quad$ Male (castrated) & $5(1)$ & $11(3)$ \\
Age (years) & $9.2 \pm 3.6(2-15)$ & $11 \pm 5.4(2-21)$ \\
Body weight (kg) & $9.8 \pm 9.3(1.7-35.0)$ & $11.5 \pm 11.7$ \\
& & $(1.2-39.4)$ \\
Body condition & $3.3 \pm 0.5(2.5-4.0)$ & $3.0 \pm 0.6(2.0-4.0)$ \\
score & &
\end{tabular}

Data are presented as mean $\pm \mathrm{SD}$ and range. $\mathrm{HD}=$ Healthy dogs, $C K D=$ Chronic kidney disease 


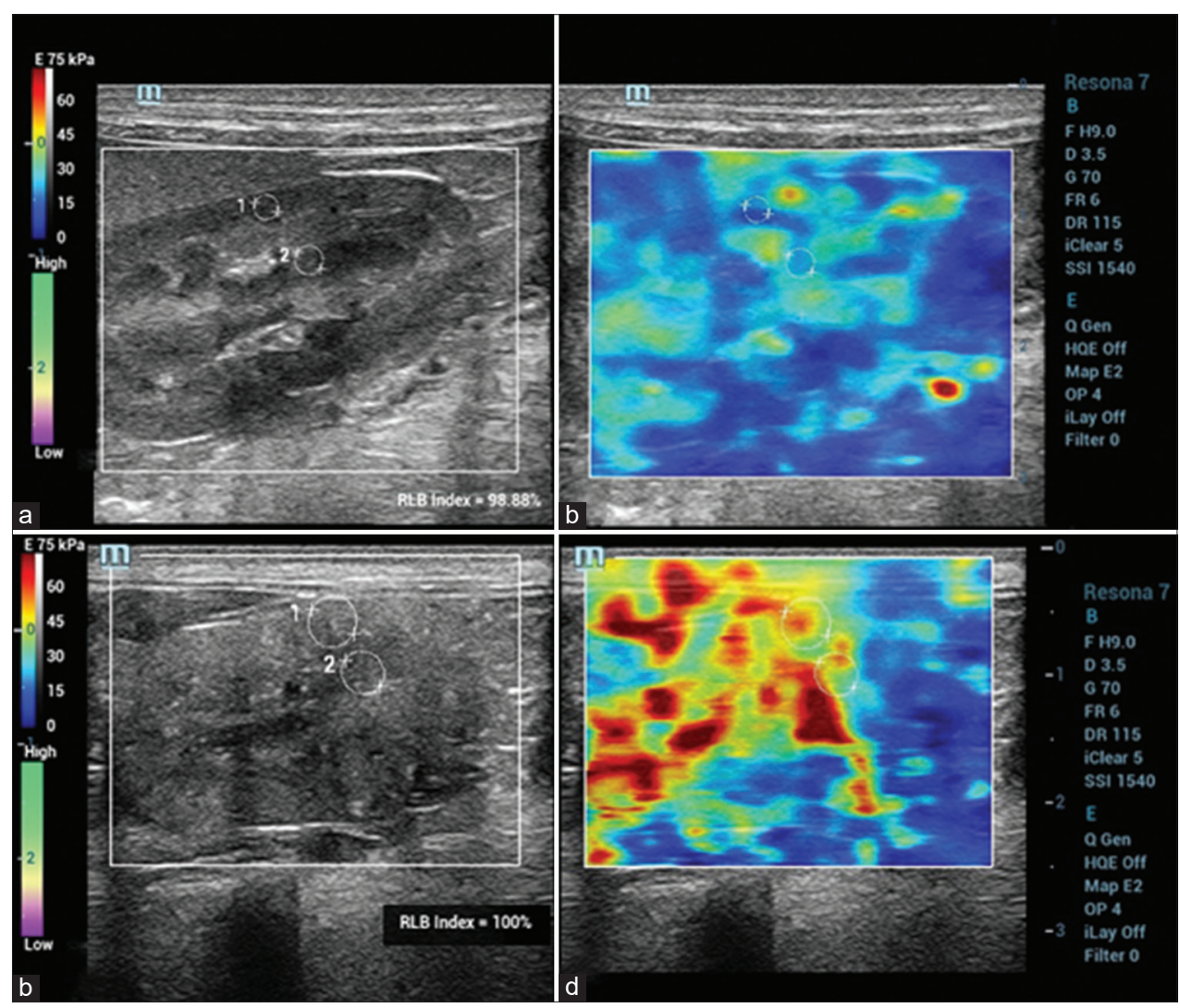

Figure-1: B-mode ultrasound images ( $a$ and $c$ ) and color quantitative elastogram superimposed on the B-mode with regions of interest ( $b$ and $d$ ) between a healthy dog ( $A$ and $B$ ) and a chronic kidney disease dog ( $c$ and $d$ ).

Pitbull Terrier (1), and Domestic Thai (2). The CKD dogs were classified as either IRIS Stage 2 (eight of $15 \mathrm{dogs}, 53.3 \%$ ) or Stage 3 (seven of $15 \mathrm{dogs}, 46.7 \%$ ).

B-mode ultrasonographic findings showed that the kidneys of dogs in the HD group had a normal shape, contour, echogenicity, and echotexture, with a mean renal length, for both right and left kidneys, of $4.0 \pm 1.0 \mathrm{~cm}$. The mean $\mathrm{K} /$ Ao ratios of the $\mathrm{HD}$ group were $7.3 \pm 1.2$ and $7.3 \pm 1.1$ for the right kidney length to aorta diameter (RK/Ao) and the left kidney length to aorta diameter (LK/Ao), respectively. In contrast, the kidneys of the dogs in the CKD group demonstrated increased cortex and/or medulla echogenicity, poor corticomedullary demarcation, and an irregular contour, with a mean renal length of $3.4 \pm 1.6$ and $4.0 \pm 1.3 \mathrm{~cm}$ for the right and the left kidneys, respectively. The mean $\mathrm{K} / \mathrm{Ao}$ ratios of CKD dogs were $5.7 \pm 2.0$ and $6.5 \pm 1.3$ for the RK/Ao and LK/Ao, respectively. The average renal lengths were not significantly different between groups. However, the average K/Ao ratios of CKD dogs were significantly lower than those of HD (RK/ Ao; $\mathrm{p}=0.005$ and LK/Ao; $\mathrm{p}=0.036$ ).

The average blood pressure of the HD group was significantly lower than that of the CKD group $(123.5 \pm 22.6 \mathrm{mmHg}$ and $141.0 \pm 22.1 \mathrm{mmHg}$, respectively, $\mathrm{p}=0.045$ ). The renal functional parameters, including the plasma concentration of Cr, BUN, USG, the UPC ratio, the Uosm/Posm ratio, and the $\mathrm{FE}_{\mathrm{Na}}, \mathrm{FE}_{\mathrm{K}}$, $\mathrm{FE}_{\mathrm{Cl}}$, and $\mathrm{FE}_{\mathrm{Mg}}$ were compared between the groups and are presented in Table-2. All renal functional parameters in the CKD group were significantly different from those in the HD group. Plasma concentrations of $\mathrm{Cr}$, BUN, and the UPC ratio of CKD dogs were significantly higher than those in $\operatorname{HD}(p<0.001)$, while USG and the Uosm/Posm ratio of CKD dogs were significantly lower than those of HDs $(p<0.001)$. The FE of electrolytes $\left(\mathrm{FE}_{\mathrm{e}}\right)$ were significantly higher in CKD dogs than in $\mathrm{HD}\left(\mathrm{FE}_{\mathrm{Na}}, \mathrm{FE}_{\mathrm{K}}, \mathrm{FE}_{\mathrm{Cl}}\right.$, and $\mathrm{FE}_{\mathrm{Mg}}$ : $\mathrm{p}<0.001$, and $\mathrm{FE}_{\mathrm{K}}: \mathrm{p}=0.001$ ).

The renal elasticity levels observed through SWE of both groups were reported as average E values calculated from either the cortex or medulla of the right and left kidneys (RK and LK). Both the average renal cortical and medullary $\mathrm{E}$ values of the right and left kidneys were not significantly difference in each group (Table-3). Both HD and CKD groups showed significantly higher of the average renal cortical E values than those of the renal medulla. In addition, when compared between groups, the average $\mathrm{E}$ values of both renal cortex and medulla in CKD dog were significantly higher than those of HD (Figure-2). Age, bodyweight, and body condition score (BCS) did not influence the average renal cortical and medullary E values of HD in this study. In addition, the average renal $\mathrm{E}$ values of cortex and medulla did not significantly differ between male and female dogs in the HD group.

The correlations between average renal $\mathrm{E}$ values and the functional renal parameters of all dogs are summarized in Table-4. The E values of both the cortex 
Table-2: Comparison of the renal functional parameters including plasma $\mathrm{Cr}$ concentrations, BUN, USG, UPC ratio, Uosm/Posm ratio, $\mathrm{FE}_{\mathrm{Na}}, \mathrm{FE}_{\mathrm{K}}, \mathrm{FE}_{\mathrm{Cl}}$ and $\mathrm{FE}_{\mathrm{Mg}}$, and the anatomical renal length parameter including $\mathrm{RK} / \mathrm{Ao}$ and $\mathrm{LK} / \mathrm{Ao}$ between the healthy dogs (HD) and chronic kidney disease dogs (CKD) and correlations between the average uPIIINP/cr ratios and those parameters.

\begin{tabular}{|c|c|c|c|c|}
\hline \multirow[t]{2}{*}{ Parameters } & \multirow[t]{2}{*}{ HD group } & \multirow[t]{2}{*}{ CKD group } & \multirow[t]{2}{*}{$\begin{array}{l}\text { Reference } \\
\text { intervals }\end{array}$} & \multirow{2}{*}{$\begin{array}{c}\text { Correlations with average } \\
\text { uPIIINP/cr ratios }\end{array}$} \\
\hline & & & & \\
\hline \multicolumn{5}{|l|}{ Renal function } \\
\hline Plasma $\mathrm{Cr}(\mathrm{mg} / \mathrm{dl})$ & $0.9 \pm 0.2 * * *$ & $2.4 \pm 1.0 * * *$ & $0.3-1.4^{* * *} *$ & $0.757 * * *$ \\
\hline BUN (mg/dl) & $20.0 \pm 6.5$ & $44.8 \pm 20.7 * * *$ & $5-21 * * *$ & $0.662 * * *$ \\
\hline $\mathrm{FE}_{\mathrm{Na}}(\%)$ & $0.2 \pm 0.2 * * *$ & $2.7 \pm 2.1 * * *$ & $<1 * * *$ & $0.858 * * *$ \\
\hline $\mathrm{FE}_{\mathrm{K}}(\%)$ & $13.3 \pm 7.1 * * *$ & $42.5 \pm 31.8^{* *}$ & $<20 * * *$ & $0.688^{* * *}$ \\
\hline $\mathrm{FE}_{\mathrm{Cl}}(\%)$ & $0.5 \pm 0.2 * * *$ & $4.2 \pm 2.6 * * *$ & $<1 * * *$ & $0.862 * * *$ \\
\hline $\mathrm{FE}_{\mathrm{Mg}}(\%)$ & $2.4 \pm 2.3^{* * *}$ & $18.4 \pm 16.7 * * *$ & $<5.4$ & $0.754 * * *$ \\
\hline USG $* * *$ & $1.045 \pm 0.01 * * *$ & $1.022 \pm 0.007 * * *$ & $1.035-1.060$ & $-0.776 * * *$ \\
\hline Uosm/Posm ratio & $4.0 \pm 1.2 *$ & $1.3 \pm 0.8 * * *$ & $\mathrm{NA} * * *$ & $-0.829 * * *$ \\
\hline UPC ratio** & $0.04 \pm 0.07 * *$ & $2.20 \pm 3.20 * * *$ & $<0.2^{\mathrm{a}}$ & 0.128 \\
\hline \multicolumn{5}{|c|}{ Anatomical renal length } \\
\hline RK/Ao & $7.3 \pm 1.2$ & $5.7 \pm 2.0 * *$ & $5.5-9.1 * *$ & $0.473 *$ \\
\hline LK/Ao & $7.3 \pm 1.1 * * *$ & $6.5 \pm 1.3 *$ & $5.5-9.1 * * * *$ & $0.411 *$ \\
\hline
\end{tabular}

aReference of UPC ratio in dogs=Non-proteinuric $(<0.2)$, borderline $(0.2-0.5)$, proteinuric $(>0.5)$. BUN=Blood urea nitrogen, $\mathrm{Cr}=$ Creatinine, $\mathrm{FE}_{\mathrm{Na}}=\mathrm{Fractional}$ excretion of sodium, $\mathrm{FE}_{\mathrm{K}}=$ Fractional excretion of potassium, $\mathrm{FE}_{\mathrm{CI}}=\mathrm{Fractional}_{\mathrm{C}}$ excretion of chloride, $\mathrm{FE}_{\mathrm{Mg}}=$ Fractional excretion of magnesium, USG=Urine specific gravity, Uosm/Posm ratio=Urine osmolality per plasma osmolality ratio, UPC ratio=Urine protein creatinine ratio, $\mathrm{RK} / \mathrm{Ao}=\mathrm{Right}$ renal length to aortic luminal diameter, $\mathrm{LK} / \mathrm{Ao}=$ Left renal length to aortic luminal diameter, uPIIINP/Cr=Urinary procollagen type III aminoterminal propeptide to creatinine ratio. Data are presented as mean \pm SD. Statistically difference between groups was made using Unpaired t-test, $* * p<0.01 ; * * * p<0.001$. Correlations between parameters were made using Spearman correlation, $* p<0.05 ; * * * p<0.001$

Table-3: The average Young's modulus $(\mathrm{E})$ values $(\mathrm{kPa})$ of renal parenchyma between HD and chronic CKD groups.

\begin{tabular}{|c|c|c|c|c|}
\hline \multirow[t]{2}{*}{ Group } & \multicolumn{2}{|c|}{ E value of LK } & \multicolumn{2}{|c|}{ E value of $R K$} \\
\hline & Cortex & Medulla & Cortex & Medulla \\
\hline HD & $27.90 \pm 4.45$ & $23.80 \pm 4.20$ & $26.81 \pm 6.95$ & $20.40 \pm 5.14$ \\
\hline CKD & $44.30 \pm 12.80$ & $35.10 \pm 10.40$ & $32.80 \pm 15.41$ & $30.25 \pm 11.25$ \\
\hline
\end{tabular}

Data are expressed as mean \pm SD. The average $\mathrm{E}$ values between left and right kidneys in each group of dog were analyzed by Paired t-test. $\mathrm{HD}=$ Healthy dogs, $\mathrm{CKD}=$ Kidney disease dogs

Table-4: The correlations between the average renal Young's modulus (E) values and plasma $\mathrm{Cr}$ concentration, BUN, USG, UPC ratio, Uosm/Posm ratio and $\mathrm{FE}_{\mathrm{Na}}, \mathrm{FE}_{\mathrm{K}}, \mathrm{FE}_{\mathrm{Cl}}$ $\mathrm{FE}_{\mathrm{Mg}}$ in clinical HD and CKD dogs.

\begin{tabular}{|c|c|c|}
\hline \multirow[t]{2}{*}{ Parameter } & $\begin{array}{l}\text { Average E values } \\
\text { of cortex }\end{array}$ & $\begin{array}{c}\text { Average E values } \\
\text { of medulla }\end{array}$ \\
\hline & $\mathbf{r}$ & $\mathbf{r}$ \\
\hline Plasma $\mathrm{Cr}$ & $0.59 * * *$ & $0.62 * * *$ \\
\hline BUN & $0.55^{* *}$ & $0.43 *$ \\
\hline USG & $-0.39 *$ & $-0.36 *$ \\
\hline UPC ratio & $0.52 * *$ & $0.51 * *$ \\
\hline Uosm/Posm ratio & $-0.41 *$ & -0.32 \\
\hline $\mathrm{FE}_{\mathrm{Na}}$ & $0.41 *$ & $0.44 *$ \\
\hline $\mathrm{FE}_{\mathrm{K}}$ & $0.40 *$ & $0.39 *$ \\
\hline $\mathrm{FE}_{\mathrm{Cl}}^{\mathrm{K}}$ & $0.42 *$ & $0.41 *$ \\
\hline $\mathrm{FE}_{\mathrm{Mg}}^{\mathrm{Cl}}$ & $0.66 * * *$ & $0.58 * * *$ \\
\hline
\end{tabular}

$\mathrm{E}$ value $=$ The Young's modulus values in kilopascal unit, $\mathrm{BUN}=$ Blood urea nitrogen, $\mathrm{Cr}=$ Creatinine, USG $=$ Urine specific gravity, UPC ratio=Urine per creatinine ratio, Uosm/Posm ratio=Urine osmolality per plasma osmolality ratio, $\mathrm{FE}_{\mathrm{Na}}=$ Fractional excretion of sodium, $\mathrm{FE}_{\mathrm{K}}=$ Fractional excretion of potassium, $\mathrm{FE}_{\mathrm{Cl}}=$ Fractional excretion of chloride, $\mathrm{FE}_{\mathrm{Mg}}=$ Fractional excretion of magnesium, $\mathrm{HD}=$ Healthy dogs, $\mathrm{CKD}=$ Chronic kidney disease. $* \mathrm{p}<0.05 ; * * \mathrm{p}<0.01 ; * * * \mathrm{p}<0.001$

and medulla showed significant positive correlation with the concentrations of plasma $\mathrm{Cr}(\mathrm{p}<0.001)$, BUN

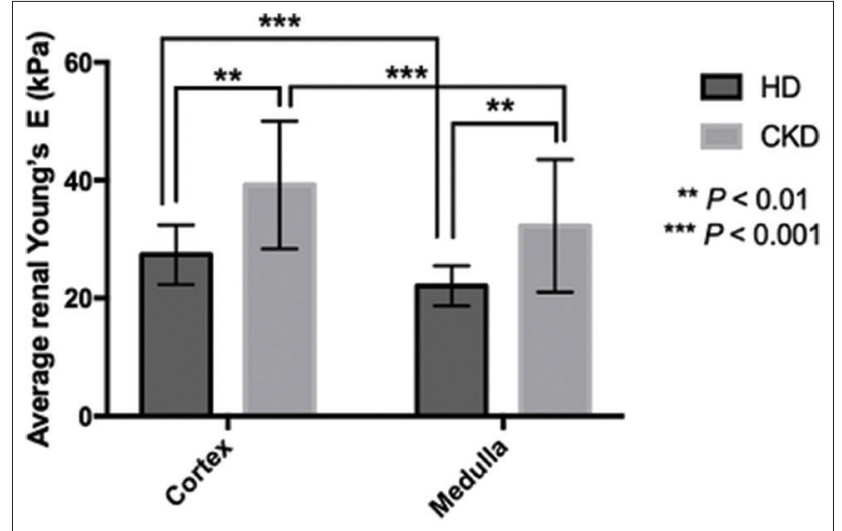

Figure-2: The average renal $E$ values of cortex and medulla (mean $\pm S D$ ) within group and between groups of dogs; the healthy dogs and chronic kidney disease group. Statistical difference between the average renal E values of cortex and medullar within each group using Paired t-test; comparison between the average renal $E$ values of cortex between groups using Unpaired t-test; comparison between the average renal $E$ values of medulla between groups using Mann-Whitney test. $* * * p<0.001 ; * * p<0.01$.

(cortex: $\mathrm{p}<0.01$; medulla; $\mathrm{p}<0.05$, respectively), UPC ratio $(p<0.01), \mathrm{FE}_{\mathrm{Na}}(\mathrm{p}<0.05), \mathrm{FE}_{\mathrm{K}}(\mathrm{p}<0.05), \mathrm{FE}_{\mathrm{Cl}}$ $(p<0.05)$, and $\mathrm{FE}_{\mathrm{Mg}}(\mathrm{p}<0.001)$ while they showed a negative correlation with USG $(\mathrm{p}<0.05)$. However, 
only the renal cortical $\mathrm{E}$ value presented a negative correlation with Uosm/Posm $(\mathrm{p}<0.05)$.

For the uPIIINP/Cr ratios, the results showed that the average $\mathrm{UPIIINP} / \mathrm{Cr}$ concentrations of CKD dogs were significantly higher than those of HD (mean uPII$\mathrm{INP} / \mathrm{Cr}$ of $3.930 \pm 3.740$ and $0.004 \pm 0.003 \mathrm{ng} / \mathrm{mgCr}$ for $\mathrm{CKD}$ and HD dogs, respectively; $\mathrm{p}<0.001$ ) (Figure-3a). The average uPIIINP/Cr ratio of CKD dogs with IRIS Stage 3 was significantly higher than that of CKD dogs with IRIS Stage 2 (mean uPIIINP/Cr of $4.468 \pm 2.026$ and $1.752 \pm 0.780 \mathrm{ng} / \mathrm{mgCr}$ for IRIS Stage 3 and IRIS Stage 2 of dogs, respectively; $p<0.01$ ) (Figure-3b). A significant positive correlation $(p<0.01)$ was found between uPIIINP/Cr ratios and average renal cortical E values (Figure-4), while uPIIINP/Cr ratios were not significantly correlated with average renal medullary E values. Moreover, the uPIIINP/Cr ratios showed positive correlations with the concentrations of plasma $\mathrm{Cr}(\mathrm{p}<0.001), \mathrm{BUN}(\mathrm{p}<0.001), \mathrm{FE}_{\mathrm{Na}}(\mathrm{p}<0.001), \mathrm{FE}_{\mathrm{K}}$ $(p<0.001), \mathrm{FE}_{\mathrm{Cl}}(\mathrm{p}<0.001)$, and $\mathrm{FE}_{\mathrm{Mg}}(\mathrm{p}<0.001)$, and the negative correlation with USG $(\mathrm{p}<0.001)$ and Uosm/Posm $(p<0.001)$ (Table-2). No significant correlation was found between the uPIIINP/Cr and either the UPC ratio or blood pressure. In addition, the uPIIINP/Cr concentrations had significant negative correlations with both RK/Ao $(\mathrm{p}<0.05)$ and LK/Ao $(\mathrm{p}<0.05)($ Table-2).

\section{Discussion}

As knowledge is updated, it has been confirmed that the principle pathological alteration underlying the CKD advancement is renal fibrosis including glomerular and tubulointerstitial fibrosis. These impairments can eventually drive the progression of kidney diseases into end-stage renal disease (ESRD) $[16,47]$. Importantly, tubulointerstitial fibrosis is the strongest indicator of CKD progression [16,17]. At present, renal biopsy is the gold standard method for achieving a final diagnosis of renal fibrosis $[16,28,48]$. However, renal biopsy is an invasive procedure with several complications, most importantly, renal hemorrhage [49]. Therefore, the novel and non-invasive techniques or biomarkers that can represent morphological tissue alterations at early stages and can indicate an association with renal function impairment would be meaningful in predicting renal functional loss and disease progression in CKD dogs.

Ultrasonographic elastography is a diagnostic imaging modality that has been applied in human medicine for a few decades. This technique can differentiate pathological tissue from normal tissue by detecting the tissue elasticity; elasticity normally decreases through pathological alterations and becomes stiff especially due to fibrotic processes such as renal fibrosis in CKD patients [50]. Ultrasonographic elastography has been employed to assist in early CKD diagnoses and in detecting renal fibrosis progression of humans [36,37,50,51]. In veterinary medicine, studies involving this technique have been continuously increasing; several studies have been published concerning the evaluation of normal kidneys in HD [43,52] and cats [53], canine mammary tumor predictions [54,55], and comparisons between benign and malignant lymph nodes in dogs and cats [56] However, the information about the renal elastography has been reported only in cats with CKD [32]. It has not been any previously reported in CKD dogs.

SWE is the latest ultrasonographic elastography technique and it has several advantages over another technique called as strain elastography (SE). For example, SWE offers real-time monitoring of both structural and tissue stiffness information through a color quantitative elastogram overlaid on a B-mode image [57,58]. Moreover, the reproducibility of SWE between observers is also excellent [59]. Therefore, ultrasonographic elastography can effectively be used for non-invasive renal fibrosis detection and monitoring. Its use might reduce the need for renal biopsy procedures in humans [59]. As previous studies were conducted in humans, the finding in this study using SWE to investigate canine renal parenchyma elasticity in CKD dogs might assist the practitioners in the diagnosis and management of canine CKD.

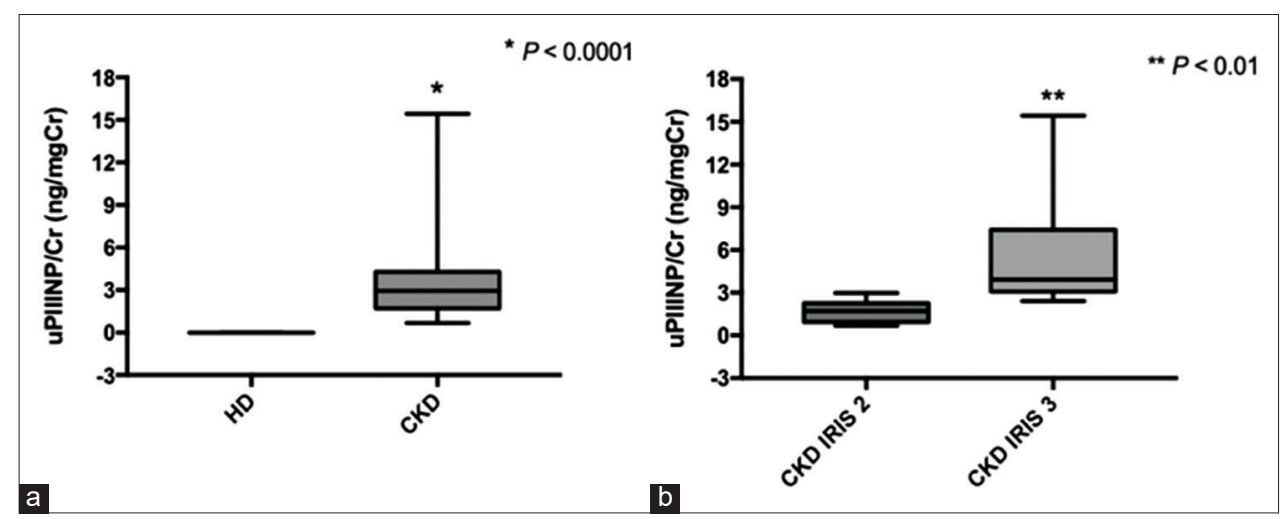

Figure-3: Box and whisker plot illustrating the uPIIINP/Cr ratios between groups using Mann-Whitney test; (a) the uPIIINP/Cr ratios between the HD and chronic kidney disease (CKD) dogs; (b) the uPIIINP/Cr ratios between CKD dogs with IRIS Stages 2 and 3. uPIIINP/Cr=Urinary procollagen type III amino-terminal propeptide to creatinine ratio, IRIS=The International Renal Interest Society staging system. 


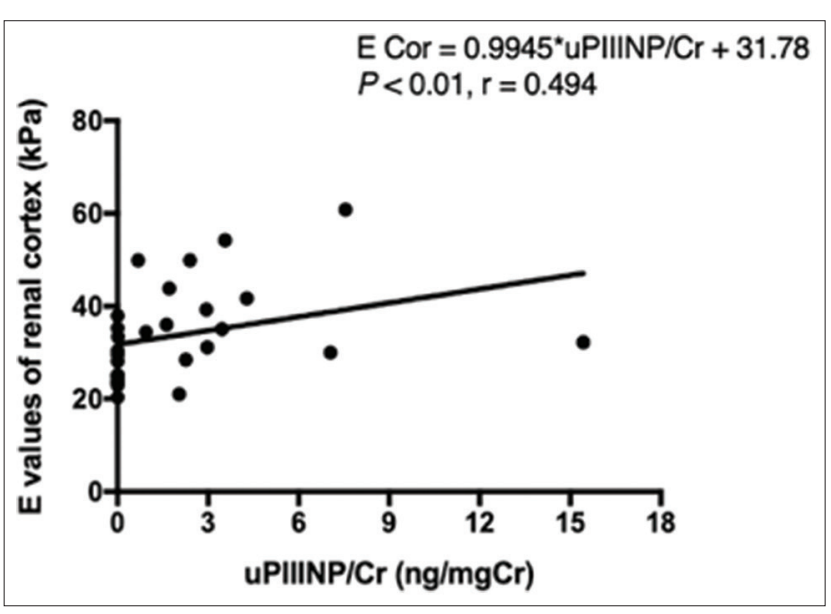

Figure-4: Correlation between average renal cortical $\mathrm{E}$ values from shear-wave elastography and uPIIINP/Cr of 30 dogs. Correlation was made using Spearman correlation coefficient. $\mathrm{E}=$ Young's modulus values, uPIIINP/Cr$=$ Urine Procollagen Type III N-Terminal Propeptide to creatinine.

In the present study, the renal length results from the B-mode ultrasonography showed that the renal length of CKD did not significantly differ from those of the HD. However, when compared the K/Ao ratio as described in the previous studied [24], both RK/Ao and $\mathrm{LK} / \mathrm{Ao}$ ratios of CKD dogs were significantly lower than those of HD. The indifferent of average renal length in between groups is likely because our study included dogs of various breeds of dogs, leading to high variability in body conformation, and renal length. Mareschal et al. [24] were reported that using $\mathrm{K} / \mathrm{Ao}$ is a suitable method for considering renal size in dogs. Our results corresponded with the previous studies in both dogs and cats [32,51,52], all of which suggest decreased that renal size is an important indicator in evaluation of CKD progression [24,51-53].

In agreement with the previous SWE study in cats [32], the renal $\mathrm{E}$ values between the right and left kidneys were not significantly different. Moreover, in both groups, our results showed that the renal cortex had the elasticity significantly lower than renal medulla which corresponds to the recent studies of using SWE to evaluate the renal elasticity in cat [32], pig [60], and also humans [37,40]. However, these results were different from the previous studied of using SE for evaluating kidneys in normal dogs [53] and cats [53]. These incongruous findings might be due to different elastography techniques being used among studies. Further comparative studies between elastography techniques should be conducted to provide more clinical data in evaluating the renal elasticity in dogs.

The renal elasticity of both the cortex and medulla in the CKD group was significantly lower than those of HD. Our findings correspond with the previous studies in both CKD cats [32] and humans $[37,41,61,62]$ in that animals and people with CKD had stiffer kidneys than those of their healthy counterparts. However, our results showed an overlapping between the average renal E values of HD dogs and CKD dogs; this might currently limit SWE use as the definitive diagnosis tool for CKD. Accordingly, SWE should be used as a supplementary tool to acquire more accurate information for detecting and monitoring of canine CKD. Furthermore, SWE may assist practitioners in diagnosing and planning the proper treatment of renal disease in subclinical cases.

Age, gender, breed, bodyweight, and BCS had no significant effect on the renal $E$ values of either cortex or medulla in this study, consistent with the previous findings in canine [43], feline [32], and human [63]. However, the small number of animals included in this study is one of the important limitations in evaluating the influence of demographic variables on SWE.

It is commonly accepted that renal tubulointerstitial fibrosis is the main final pathway of kidney disease [47] that is highly correlated with renal functional deterioration in humans [64,65], dogs [66], and cats $[66,67]$. In the present study, renal tubular functions were evaluated through the Uosm/Posm ratio, $\mathrm{FE}_{\mathrm{Na}}, \mathrm{FE}_{\mathrm{K}}, \mathrm{FE}_{\mathrm{Cl}}$, and $\mathrm{FE}_{\mathrm{Mg}}$ as a reflection of renal absorption and excretion of water and electrolytes. CKD dogs in this study presented aberrant urinary concentrating ability, contributing to the lower urine osmolality and Uosm/Posm ratio than those in HD. These results correspond with the findings of a previous study in cats [32], in which cats with CKD had lower urine osmolality and Uosm/Posm ratio than healthy cats. In addition, it has been reported that $\mathrm{FE}_{\mathrm{e}}$ is a highly sensitive parameter for the assessment of renal tubular impairment in dogs with CKD [45]. Moreover, a study in humans revealed that $\mathrm{FE}_{\mathrm{Mg}}$ showed a positive correlation with the degree of tubulointerstitial fibrosis; thus, it may be a useful marker for identifying the severity of renal tubular cell damage, especially of the renal proximal tubule [68].

For the relationships between the renal parenchyma elasticity and renal function parameters, the results demonstrate that the renal cortical $\mathrm{E}$ values or renal cortical elasticity was more significantly correlated with the renal functions than the renal $\mathrm{E}$ values of the medulla. In renal cortical elasticity correlations, the significantly positive correlations were observed with concentrations of plasma $\mathrm{Cr}$, BUN, the $\mathrm{UPC}$ ratio, $\mathrm{FE}_{\mathrm{Na}}, \mathrm{FE}_{\mathrm{K}}, \mathrm{FE}_{\mathrm{Cl}}$, and $\mathrm{FE}_{\mathrm{Mg}}$. Meanwhile, the significantly negative correlations were observed between the renal cortical elasticity and either USG or the Uosm/Posm ratio. The results correspond with the prior studies in human [36] and also in cats [32] that decreasing renal elasticity in CKD patients was associated with progressive deteriorations of renal functions. These findings suggest that the renal cortical $E$ value is more highly correlated with renal function compared with the renal medullary E value; this is consistent with a previous study in cats [32]. This may be because the renal cortex contains both the glomeruli, which are responsible for plasma filtration [69], and the proximal tubules, which are responsible for 
the reabsorption and secretion of substances, particularly of water and electrolytes [70].

Although urine osmolality and $\mathrm{FE}_{\mathrm{e}}$ can represent the absorption and excretion capability of water and electrolytes, the results can be altered by many exogenous and endogenous factors, particularly diet, and hydration status [70]. To decrease these confounding effects in this study, we strictly evaluated the hydration status of all dogs by taking a complete history and performing physical examinations; only dogs with normal hydration were included in this study. However, the influence of food on these parameters could not be controlled in this study, because the use of a prescription diet is the clinical guidelines for CKD management. Therefore, this might be an additional limitation of this study.

The present study investigated the correlations of uPIIINP/Cr with the renal $\mathrm{E}$ values and the renal functional parameters due to the previous evidence indicating that $\mathrm{uPIIINP} / \mathrm{Cr}$ ratio correlated with the severity of renal functional impairment and the degree of renal fibrosis in humans [16,29-31]. Moreover, uPIIINP levels also correlated with urinary levels of transforming growth factor beta 1 (TGF- $\beta 1$ ) [30], which is commonly used as biomarker for renal fibrosis, assessment in humans and cats [71]. However, urinary TGF- $\beta 1$ had no significant association with renal function impairment in either humans [48] or cats [72]. In humans, elevated uPIIINP levels could signify deteriorating renal tubular reabsorption ability due to renal fibrosis in CKD patients [16,29,30]. Moreover, a recent study showed that the uPIIINP/Cr ratio had a significant positive correlation with decreased renal parenchymal elasticity as detected by SWE in cats with CKD [32].

The uPIIINP/Cr ratios were significantly higher in CKD dogs than in HD, in agreement with the previous studies of CKD in humans [16,29,30], cats [32], and dogs with cardiac remodeling [33], idiopathic pulmonary fibrosis [34], and liver fibrosis [35]. In addition, the results showed no effects regarding the degree of proteinuria on the UPIIINP level in canine CKD similar to the previous studies in humans [30] and cats [32]. This maintains that there is no significant correlation between the $\mathrm{uPIIINP} / \mathrm{Cr}$ ratio and UPC in those species.

Moreover, we also found that the average uPIIINP/Cr ratios were significantly higher in CKD dogs with IRIS Stage 3 than those in CKD dogs with IRIS Stage 2, with little overlap of the average uPIIINP/Cr ratios. This indicates that the uPIIINP/Cr ratio might be a good biomarker to use as an adjunctive tool for detecting the progression of CKD in dogs. Nevertheless, we did not include all CKD IRIS stages in this study, as it was difficult to categorize dogs with Stage 1 CKD by Cr only, and there were few Stage 4 CKD dogs; also some were not in a stable condition due to their poor physical health, especially if they had progressed to ESRD. Thus, recruiting dogs with all IRIS stages are challenging and may require the use of more laboratory parameters, such as serum or plasma symmetric dimethylarginine (SDMA) to differentiate IRIS Stages 1 and 2 [44].

In agreement with a previous feline study [32], a statistically positive correlation was found between the $\mathrm{uPIIINP} / \mathrm{Cr}$ ratio and the average renal cortical $\mathrm{E}$ values which suggest that the renal cortical $E$ value might be a promising indicator to assist in the evaluation of renal fibrosis in canine CKD.

In addition, the uPIIINP/Cr ratios had significantly positive correlations with the concentrations of plasma $\mathrm{Cr}, \mathrm{BUN}, \mathrm{FE}_{\mathrm{Na}}, \mathrm{FE}_{\mathrm{K}}, \mathrm{FE}_{\mathrm{C},}$ and $\mathrm{FE}_{\mathrm{Mg}}$, but showed significantly negative correlations with USG and Uosm/Posm. These results demonstrated that high uPIIINP/Cr ratios were associated with both glomerular and tubular renal functional deterioration. Our findings correspond with those of a study in humans with CKD reporting that uPIIINP correlated with blood $\mathrm{Cr}$ levels and the estimated GFR [16]. Moreover, uPII$\mathrm{INP} / \mathrm{Cr}$ correlated strongly with $\mathrm{FE}_{\mathrm{Ng}}, \mathrm{FE}_{\mathrm{C}}$, and $\mathrm{FE}_{\mathrm{Mg}}$, all of which are associated with proximal renal tubular function, and also had strong correlations with USG and Uosm/Posm, which are associated with a kidney's ability to concentrate the urine.

At present, in veterinary medicine, information concerning renal function parameters and renal elasticity in dogs have not yet been reported. Moreover, the correlations between the uPIIINP/Cr ratio and renal elasticity in canine CKD have not been evaluated. This study is the first report of renal elasticity observed by SWE and the uPIIINP/Cr in CKD dogs. This information would be useful for clinical practitioners and may be beneficial for further studies.

Aside from the aforementioned dietary limitations, a main limitation of this study is that renal histopathology was not investigated in the comparison of renal elasticity determined by SWE and uPIIINP/Cr ratios due to the impracticality and invasiveness of renal biopsies. Furthermore, the small number of included dogs in the study was not adequate to determine the influence of demographic variables on SWE.

\section{Conclusion}

SWE can be utilized as an additional diagnostic imaging tool for evaluating renal elasticity in CKD dogs since elasticity is significantly correlated with renal functional parameters. The renal cortex had lower elasticity than the renal medulla in both $\mathrm{HD}$ and CKD groups; also, the kidneys of dogs in the CKD group were stiffer than those of HD in this study. In addition, the renal cortical $\mathrm{E}$ values were significantly correlated with the uPIIINP/Cr ratios. Furthermore, the $\mathrm{uPIIINP} / \mathrm{Cr}$ ratios were higher in CKD dogs. Therefore, the uPIIINP/Cr might also be a promising biomarker for a complementary evaluation of renal fibrosis in canine CKD. However, further studies are needed to evaluate the clinical E value of SWE for evaluating and monitoring dogs with CKD and to 
explore the relationship between uPIIINP/Cr ratios and different stages of renal fibrosis in dogs. In the future, SWE and uPIIINP/Cr ratios might be useful in animals, in which a renal biopsy is contraindicated.

\section{Authors' Contributions}

Study conception and design: CT, SS, CB, and $\mathrm{NC}$; acquisition of data: $\mathrm{CT}$ and $\mathrm{NC}$; laboratory test: $\mathrm{CT}$ and $\mathrm{CB}$; analysis and interpretation of data: $\mathrm{CT}$, $\mathrm{SS}, \mathrm{CB}$, and NC; drafting of manuscript: CT, SS and $\mathrm{NC}$; critical revision: $\mathrm{CT}, \mathrm{SS}, \mathrm{CB}$, and $\mathrm{NC}$; all authors read and approved the final manuscript.

\section{Acknowledgments}

We would like to thank you the all technical recommendations from Mindray Medical (Thailand). This study was granted from the $100^{\text {th }}$ Anniversary Chulalongkorn University for Doctoral Scholarship and the $90^{\text {th }}$ Anniversary of Chulalongkorn University Fund (Ratchadaphiseksomphot Endowment Fund), Grant number GCUGR1125613085D, the overseas Research Experience Scholarship for Graduate Student, Graduate School of Chulalongkorn University and Faculty of Veterinary Science, Chulalongkorn University. The funders supported the data collection, laboratory equipment, and analysis and publication fee.

\section{Competing Interests} interests.

The authors declare that they have no competing

\section{Publisher's Note}

Veterinary World remains neutral with regard to jurisdictional claims in published institutional affiliation.

\section{References}

1. Polzin, D.J. (2014) Chronic kidney disease in small animals. Vet. Clin. Small Anim. Pract., 41(1): 15-30.

2. Guidi, G., Rossini, C., Cinelli, C., Meucci, V. and Lippi, I. (2012) Canine chronic kidney disease: Retrospective study of a 10-year period of clinical activity. In: Veterinary Science, Current Aspects in Biology, Animal Pathology, Clinic and Food Hygiene. Springer, Verlag Berlin Heidelberg. p115-118.

3. Foster, J.D. (2013) Canine Chronic Kidney Disease. Current Diagnostics and Goals for Long-Term Management Today's Veterinary Practice. p21-26.

4. O’Neill, D.G., Elliott, J., Church, D.B., McGreevy, P.D., Thomson, P.C. and Brodbelt, D.C. (2013) Chronic kidney disease in dogs in UK veterinary practices: Prevalence, risk factors, and survival. J. Vet. Intern. Med., 27(4): 814-821.

5. Brown, S.A. (2007) Management of chronic kidney disease. In: Elliot, J., editor. BSAVA Manual of Canine and Feline Nephrology and Urology. $2^{\text {nd }}$ ed. British Small Animal Veterinary Association, Gloucester. p223-230.

6. Macdougall, D.F., Cook, T., Steward, A.P. and Cattell, V. (1986) Canine chronic renal disease: Prevalence and types of glomerulonephritis in the dog. Kidney Int., 29(6): 1144-1151.

7. Sosnar, M., Kohout, P., Růžička, M. and Vrbasova, L. (2003) Retrospective study of renal failure in dogs and cats admitted to University of Veterinary and Pharmaceutical
Sciences, Brno during 1999-2001. Acta Vet. Brno, 72(4): 593-598.

8. Vaden, S.L. (2011) Glomerular disease. Top. Companion Anim. Med., 26(3): 128-134.

9. DiBartola, S.P., Tarr, M.J., Webb, D.M. and Giger, U. (1990) Familial renal amyloidosis in Chinese Shar-Pei dogs. J. Am. Vet. Med. Assoc., 197(4): 483-487.

10. Jones, B.R., Gething, M.A., Badcoe, L.M., Pauli, J.V. and Davies, E. (1989) Familial progressive nephropathy in young Bull Terriers. N. Z. Vet. J., 37(2): 79-82.

11. Lees, G.E., Helman, R.G., Homco, L.D., Millichamp, N.J., Hunter, J.F. and Frey, M.S. (1998) Early diagnosis of familial nephropathy in English cocker spaniels. J. Am. Anim. Hosp. Assoc., 34(3): 189-195.

12. McAloose, D., Casal, M., Patterson, D.F. and Dambach, D.M. (1998) Polycystic kidney and liver disease in two related West Highland White Terrier litters. Vet. Pathol., 35(1): 77-80.

13. Chandler, M.L., Elwood, C., Murphy, K.F., Gajanayake, I. and Syme, H.M. (2007) Juvenile nephropathy in 37 boxer dogs. J. Small Anim. Pract., 48(12): 690-694.

14. Bartges, J.W. (2012) Chronic kidney disease in dogs and cats. Vet. Clin. Small Anim. Pract., 42(4): 669-692.

15. Lee, S.Y., Kim, S.I. and Choi, M.E. (2015) Therapeutic targets for treating fibrotic kidney diseases. Transl. Res., 165(4): 512-530.

16. El Ghoul, B., Squalli, T., Servais, A., Elie, C., MeasYedid, V., Trivint, C., Vanmassenhove, J., Gru nfeld, J.P., Olivo-Marin, J.C., Thervet, E., Noel, L.H., Prie, D. and Fakhouri, F. (2010) Urinary procollagen III aminoterminal propeptide (PIIINP): A fibrotest for the nephrologist. Clin. J. Am. Soc. Nephrol., 5(2): 205-210.

17. Barnes, J.L., Ii, W.F.G. (2011) Renal interstitial fibrosis: A critical evaluation of the origin of myofibroblasts. Contrib. Nephrol., 169: 73-93.

18. Lees, G.E. (2004) Early diagnosis of renal disease and renal failure. Vet. Clin. Small. Anim. Pract., 34(4): 867-885.

19. Grauer, G.F. (2005) Early detection of renal damage and disease in dogs and cats. Vet. Clin. Small Anim. Pract., 35(3): 581-596.

20. Tenhündfeld, J., Wefstaedt, P. and Nolte, I.J.A. (2009) A randomized controlled clinical trial of the use of benazepril and heparin for the treatment of chronic kidney disease in dogs. J. Am. Vet. Med. Assoc., 234(8): 1031-1037.

21. Cobrin, A.R., Blois, S.L., Kruth, S.A., Abrams-Ogg, A.C.G. and Dewey, C. (2013) Biomarkers in the assessment of acute and chronic kidney diseases in the dog and cat. $J$. Small Anim. Pract., 54(12): 647-655.

22. Hall, J.A., Yerramilli, M., Obare, E., Yerramilli, M., Almes, K. and Jewell, D.E. (2016) Serum concentrations of symmetric dimethylarginine and creatinine in dogs with naturally occurring chronic kidney disease. J. Vet. Intern. Med., 30(3): 794-802.

23. Dahlem, D.P., Neiger, R., Schweighauser, A., Francey, T., Yerramilli, M., Obare, E. and Steinbach, S.M.L. (2017) Plasma symmetric dimethylarginine concentration in dogs with acute kidney injury and chronic kidney disease. J. Vet. Intern. Med., 31(3): 799-804.

24. Mareschal, A., D'Anjou, M.A., Moreau, M., Alexander, K. and Beauregard, G. (2007) Ultrasonographic measurement of kidney-to-aorta ratio as a method of estimating renal size in dogs. Vet. Radiol. Ultrasound., 48(5): 434-438.

25. Hecht, S. and Henry, G.A. (2011) Ultrasonography of the urinary tract. In: Bartges, J. and Polzin, D.J., editors. Textbook of Nephrology and Urology of Small Animals. Blackwell Publishing, USA. p128-145.

26. Farris, A.B. and Alpers, C.E. (2014) What is the best way to measure renal fibrosis?: A pathologist's perspective. Kidney Int. Suppl., 4(1): 9-15.

27. Wong, M.G. and Pollock, C.A. (2014) Biomarkers in kidney fibrosis: Are they useful? Kidney Int. Suppl., 4(1): 79-83.

28. Vaden, S.L. (2005) Renal biopsy of dogs and cats. Clin. 
Tech. Small Anim. Pract., 20(1): 11-22.

29. Soylemezoglu, O., Wild, G., Dalley, A.J., MacNeil, S., Milford-Ward, A., Brown, C.B. and El Nahas, A.M. (1997) Urinary and serum Type III collagen: Markers of renal fibrosis. Nephrol. Dial. Transplant., 12(9): 1883-1889.

30. Teppo, A.M., Törnroth, T., Honkanen, E. and GrönhagenRiska, C. (2003) Urinary amino-terminal propeptide of Type III procollagen (PIIINP) as a marker of interstitial fibrosis in renal transplant recipients. Transplantation, 75(12): 2113-2119.

31. Ix, J.H., Biggs, M.L., Mukamal, K., Djousse, L., Siscovick, D., Tracy, R., Katz, R., Delaney, J.A., Chaves, P., Rifkin, D.E., Hughes-Austin, J.M., Garimella, P.S., Sarnak, M.J., Shlipak, M.G. and Kizer, J.R. (2015) Urine collagen fragments and CKD progression the cardiovascular health study. J. Am. Soc. Nephrol., 26(10): 2494-2503.

32. Thanaboonnipat, C., Sutayatram, S., Buranakarl, C. and Choisunirachon, N. (2019) Renal shear wave elastography and urinary procollagen Type III amino-terminal propeptide (uPIIINP) in feline chronic kidney disease. BMC Vet. Res., 15(1): 54

33. Glińska-Suchocka, K., Orłowska, A., Jankowski, M., Kubiak, K. and Spużak, J. (2016) Serum concentrations of PIIINP aminopeptide in dogs with liver fibrosis. Pol. J. Vet. Sci., 19(2): 365-369.

34. Heikkilä, H.P., Krafft, E., Jespers, P., McEntee, K., Rajamäki, M.M. and Clercx, C. (2013) Procollagen Type III amino terminal propeptide concentrations in dogs with idiopathic pulmonary fibrosis compared with chronic bronchitis and eosinophilic bronchopneumopathy. Vet. J., 196(1): 52-56.

35. Hezzell, M.J., Boswood, A., Chang, Y.M., Moonarmart, W. and Elliott, J. (2012) Associations among serum N-terminal procollagen Type III concentration, urinary aldosterone-to-creatinine ratio, and ventricular remodeling in dogs with myxomatous mitral valve disease. Am. J. Vet. Res., 73(11): 1765-1774.

36. Lin, H.Y., Lee, Y.L., Lin, K.D., Chiu, Y.W., Shin, S.J., Hwang, S.J., Chen, H.C. and Hung, C.C. (2017) Association of renal elasticity and renal function progression in patients with chronic kidney disease evaluated by real-time ultrasound elastography. Sci. Rep., 7: 43303.

37. Peng, L., Zhong, T., Fan, Q., Liu, Y., Wang, X. and Wang, L. (2017) Correlation analysis of renal ultrasound elastography and clinical and pathological changes in patients with chronic kidney disease. Clin. Nephrol., 87(6): 293-300.

38. Bragato, N., Borges, N.C. and Fioravanti, M.C.S. (2017) B-mode and Doppler ultrasound of chronic kidney disease in dogs and cats. Vet. Res. Commun., 41(4): 307-315.

39. Hart, D.V., Winter, M.D., Conway, J. and Berry, C.R. (2013) Ultrasound appearance of the outer medulla in dogs without renal dysfunction. Vet. Radiol. Ultrasound., 54(6): 652-658.

40. Grenier, N., Gennisson, J.L., Cornelis, F., Le Bras, Y. and Couzi, L. (2013) Renal ultrasound elastography. Diagn. Interv. Imaging, 94(5): 545-550.

41. Guo, L.H., Xu, H.X., Fu, H.J., Peng, A., Zhang, Y.F. and Liu, L.N. (2013) Acoustic radiation force impulse imaging for noninvasive evaluation of renal parenchyma elasticity: Preliminary findings. PLoS One, 8(7): e68925.

42. Donald, R.A. and Pradeep, P.P. (2004) The Science and Engineering of Materials. $2^{\text {nd }}$ ed. Thomson learning Press, Stamford. p234-257.

43. Holdsworth, A., Bradley, K., Birch, S., Browne, W.J. and Barberet, V. (2014) Elastography of the normal canine liver, spleen and kidneys. Vet. Radiol. Ultrasound, 55(6): 620-627.

44. International Renal Interest Society. (2013) Elanco Animal Health. CKD Staging in Cats. Available from: http://www.iris-kidney.com/pdf/IRIS_2017_Staging_of CKD_09May18.pdf. Retrieved on 28-01-2019.

45. Buranakarl, C., Ankanaporn, K., Thammacharoen, S. and Trisirisoj, M. (2007) Relationships between degree of azotaemia and blood pressure, urinary protein: Creatinine ratio and fractional excretion of electrolytes in dogs with renal azotaemia. Vet. Res. Commun., 31(3): 245-257.

46. Franchi-Abella, S., Elie, C. and Correas, J.M. (2013) Ultrasound elastography: Advantages, limitations and artifacts of the different techniques from a study on a phantom. Diagn. Interv. Imaging, 94(5): 497-501.

47. Prunotto, M., Ghiggeri, G., Bruschi, M., Gabbiani, G., Lescuyer, P., Hocher, B., Chaykovska, L., Berrera, M. and Moll, S. (2011) Renal fibrosis and proteomics: Current knowledge and still key open questions for proteomic investigation. J. Proteomics, 74(10): 1855-1870.

48. Mansour, S.G., Puthumana, J., Coca, S.G., Gentry, M. and Parikh, C.R. (2017) Biomarkers for the detection of renal fibrosis and prediction of renal outcomes: A systematic review. BMC Nephrol., 18(1): 72.

49. Farris, A.B. and Colvin, R.B. (2012) Renal Interstitial fibrosis: Mechanisms and evaluation. Curr. Opin. Nephrol. Hypertens., 21(3): 289-300.

50. Berchtold, L., Friedli, I., Vallée, J.P., Moll, S., Martin, P.Y. and Seigneux, S.D. (2017) Diagnosis and assessment of renal fibrosis: The state of the art. Swiss Med. Wkly., 147: w14442.

51. Hassan, K., Loberant, N., Abbas, N., Fadi, H., Shadia, H. and Khazim, K. (2016) Shear wave elastography imaging for assessing the chronic pathologic changes in advanced diabetic kidney disease. Ther. Clin. Risk Manag., 12: 1615-1622.

52. Jeon, S., Lee, G., Lee, S.K., Kim, H., Yu, D. and Choi, J. (2015) Ultrasonographic elastography of the liver, spleen, kidneys, and prostate in clinically normal beagle dogs. Vet. Radiol. Ultrasound, 56(4): 425-431.

53. White, J., Gay, J., Farnsworth, R., Mickas, M., Kim, K. and Mattoon, J. (2014) Ultrasound elastography of the liver, spleen, and kidneys in clinically normal cats. Vet. Radiol. Ultrasound, 55(4): 428-434.

54. Feliciano, M.A.R., Ramirez, R.A.U., Maronezi, M.C., Maciel, G.S., Avante, M.L., Senhorello, I.L.S., Mucédola, T., Gasser, B., Carvalho, C.F. and Vicente, W.R.R. (2018) Accuracy of four ultrasonography techniques in predicting histopathological classification of canine mammary carcinomas. Vet. Radiol. Ultrasound, 59(5): 444-452.

55. Feliciano, M.A.R., Uscategui, R.A.R., Maronezi, M.C., Simões, A.P.R., Silva, P., Gasser, B., Pavan, L., Carvalho, C.F., Canola, J.C. and Vicente, W.R.R. (2017) Ultrasonography methods for predicting malignancy in canine mammary tumors. PLoS One, 12(5): e0178143.

56. Seile, G.S. and Griffith, E. (2018) Comparisons between elastographic stiffness scores for benign versus malignant lymph nodes in dogs and cats. Vet. Radiol. Ultrasound, 59(1): 79-88

57. Ferraioli, G., Tinelli, C., Dal Bello, B., Zicchetti, M., Filice, G. and Filice, C. (2012) Accuracy of real-time shear wave elastography for assessing liver fibrosis in chronic hepatitis C: A pilot study. Hepatology, 56(6): 2125-2133.

58. Cosgrove, D., Piscaglia, F., Bamber, J., Bojunga, J., Correas, J.M., Gilja, O.H., Klauser, A.S., Sporea, I., Calliada, F., Cantisani, V., D’Onofrio, M., Drakonaki, E.E., Fink, M., Friedrich-Rust, M., Fromageau, J., Havre, R.F., Jenssen, C., Ohlinger, R., Săftoiu, A., Schaefer, F. and Dietrich, C.F. (2012) EFSUMB guidelines and recommendations on the clinical use of ultrasound elastography. Part 2: Clinical applications. Ultraschall. Med., 34(3): 238-253.

59. Anvari, A., Barr, R.G., Dhyani, M. and Samir, A.E. (2015) Clinical application of sonoelastography in thyroid, prostate, kidney, pancreas, and deep venous thrombosis. Abdom. Imaging, 40(4): 709-722.

60. Gennisson, J.L., Deffieux, T., Fink, M. and Tanter, M. (2013) Ultrasound elastography: Principles and techniques. Diagn. Interv. Imaging, 94(5): 487-495.

61. Hu, Q., Gou, Y., Sun, C., Ding, W., Xu, K., Gu, B., Xia, G. and Ding, Q. (2014) The prognostic value of C-reactive protein in renal cell carcinoma: A systematic review and 
meta-analysis. Urol. Oncol., 32: 50.e1-8.

62. Samir, A.E., Dhyani, M., Anvari, A., Prescott, J., Halpern, E.F., Faquin, W.C. and Stephen, A. (2015) Shearwave elastography for the preoperative risk stratification of follicular patterned lesions of the thyroid: Diagnostic accuracy and optimal measurement plane. Radiology, 277(2): 565-573.

63. Singh, H., Panta, O.B., Khanal, U. and Ghimire, R.K. (2017) Renal cortical Elastography: Normal values and variations. J. Med. Ultrasound, 25(4): 215-220.

64. Risdon, R.A., Sloper, J.C. and De Wardener, H.E. (1968) Relationship between renal function and histological changes found in renal-biopsy specimens from patients with persistent glomerular nephritis. Lancet, 292(7564): 363-366.

65. Nath, K.A. (1992) Tubulointerstitial changes as a major determinant in the progression of renal damage. Am. J. Kidney Dis., 20(1): 1-17.

66. Yabuki, A., Mitani, S., Fujiki, M., Misumi, K., Endo, Y., Miyoshi, N. and Yamoto, O. (2010) Comparative study of chronic kidney disease in dogs and cats: Induction of myofibroblasts. Res. Vet. Sci., 88(2): 294-299.

67. Chakrabarti, S., Syme, H.M., Brown, C.A. and Elliott, J.
(2013) Histomorphometry of feline chronic kidney disease and correlation with markers of renal dysfunction. Vet. Pathol., 50(1): 147-155.

68. Futrakul, P., Yenrudi, S., Futrakul, N., Sensirivatana, R., Kingwatanakul, P., Jungthirapanich, J., Cherdkiadtikul, T., Laohapaibul, A., Watana, D., Singkhwa, V., Futrakul, S. and Pongsin, P. (1999) Tubular function and tubulointerstitial disease. Am. J. Kidney Dis., 33(5): 886-891.

69. Clarkson, C.E. and Fletcher, T.F. (2011) Anatomy of the kidney and proximal ureter. In: Bartges, J. and Polzin, D.J., editors. Nephrology and Urology of Small Animals. Wiley, Chichester. p1-9.

70. DiBartola, S.P. (2012) Applied renal physiology. In: Di Bartola, S.P., editor. Fluid, Electrolyte, and Acid-Base Disorders in Small Animal Practice. $4^{\text {th }}$ ed. Elsevier, St Louis. p2-43.

71. Lawson, J.S., Syme, H.M., Wheeler-Jones, C.P.D. and Elliott, J. (2016) Urinary active transforming growth factor $\beta$ in feline chronic kidney disease. Vet. J., 214: 1-6.

72. Lawson, J., Elliott, J., Wheeler-Jones, C., Syme, H. and Jepson, R. (2015) Renal fibrosis in feline chronic kidney disease: Known mediators and mechanisms of injury. Vet. J., 203(1): 18-26. 